\title{
Review of: "Standard (3, 5)-threshold quantum secret sharing by maximally entangled 6 -qubit states"
}

\section{KARTICK SUTRADHAR}

Potential competing interests: The author(s) declared that no potential competing interests exist.

This paper is technically sound and well organized. 